\title{
Amplitude modulation in $\delta$ Sct stars: statistics from an ensemble of Kepler targets
}

\author{
Dominic M. Bowman ${ }^{1, \star}$, Donald W. Kurtz ${ }^{1}$, Michel Breger ${ }^{2}$, Simon J. Murphy ${ }^{3,4}$, and Daniel L. Holdsworth ${ }^{1}$ \\ ${ }^{1}$ Jeremiah Horrocks Institute, University of Central Lancashire, Preston PR1 2HE, UK \\ ${ }^{2}$ Department of Astronomy, University of Texas, Austin, TX 78712, USA \\ ${ }^{3}$ Sydney Institute for Astronomy (SIfA), School of Physics, The University of Sydney, NSW 2006, Australia \\ ${ }^{4}$ Stellar Astrophysics Centre, Department of Physics and Astronomy, Aarhus University, DK-8000 Aarhus C, Denmark
}

\begin{abstract}
The results of a search for amplitude modulation of pulsation modes in $983 \delta$ Sct stars, which have effective temperatures between $6400 \leq T_{\text {eff }} \leq 10000 \mathrm{~K}$ in the Kepler Input Catalogue and were continuously observed by the Kepler Space Telescope for $4 \mathrm{yr}$, are presented. A total of $603 \delta$ Sct stars (61.3 per cent) are found to exhibit at least one pulsation mode that varies significantly in amplitude over 4 yr. Furthermore, it is found that amplitude modulation is not restricted to a specific region within the classical instability strip in the HR diagram, therefore its cause is not necessarily dependent on stellar parameters such as $T_{\text {eff }}$ or $\log g$. On the other hand, many $\delta$ Sct stars show constant pulsation amplitudes demonstrating that the cause of pulsational non-linearity in these stars is not well understood.
\end{abstract}

\section{Introduction}

The multiperiodic pulsators known as $\delta$ Sct stars are the most common group of variable $\mathrm{A}$ and $\mathrm{F}$ stars, and are found at the intersection of the classical instability strip and the main sequence in the HR diagram. On the main sequence, $\delta$ Sct stars typically range from A2 to F2 in spectral type [8] and within the effective temperature range of $6300 \leq T_{\text {eff }} \leq 8600 \mathrm{~K}[10]$. Pulsations in $\delta$ Sct stars are excited by the $\kappa$-mechanism operating in the He II ionisation zone, which produces low radial order pressure (p) modes. Typical pulsation periods observed in $\delta$ Sct stars are of order a few hours, but can be as short as $15 \mathrm{~min}$ [10]. The Kepler Space Telescope provided a vast increase in the number of $\delta$ Sct stars observed at a high photometric precision and high duty cycle over a time span of $4 \mathrm{yr}$. In this article, the results from a search for amplitude modulation and non-linearity using $983 \delta$ Sct stars that were continuously observed by Kepler for $4 \mathrm{yr}$ are presented [4].

There are different mechanisms that can cause nonlinearity in a pulsating star. For example, combination frequencies are mathematical sum and difference frequencies of pulsation mode frequencies, $v_{i}$ and $v_{j}$, that have the form $n v_{i} \pm m v_{j}$, where $n$ and $m$ are integers. Possible mechanisms to explain combination frequencies are that the stellar medium does not respond linearly to a pulsation wave, or that the dependence of emergent flux variation is not a linear transformation from the temperature variation $\left(F=\sigma T^{4}\right)$, which are often grouped into what is termed a non-linear distortion model [11]. These non-linear effects differ to families of pulsation modes that are resonantly

\footnotetext{
^e-mail: dmbowman@uclan.ac.uk
}

excited due to the coupling of modes inside a star [5, 6]. This form of pulsational non-linearity gives rise to variable frequencies and amplitudes in pulsation modes over time, which will appear as a cluster of unresolved peaks in an amplitude spectrum if the length of observations is shorter than the modulation cycle [6].

\subsection{Mode coupling in $\delta$ Sct stars}

Coupled pulsation modes are grouped into a family containing one child mode with frequency $v_{1}$, and two parent modes with frequencies $v_{2}$ and $v_{3}$, which satisfy a frequency resonance condition $[5,6]$ of

$$
v_{1} \simeq v_{2} \pm v_{3}
$$

but must also satisfy the phase relation of

$$
\phi_{1}=\phi_{2} \pm \phi_{3} \text {. }
$$

However, equations 1 and 2 do not distinguish if $v_{1}$ is a resonantly-excited pulsation mode frequency, or is a combination frequency that describes the non-linear interaction of the parent pulsation modes. To make this distinction, the amplitude of a child mode can be modelled as a product of the two parent mode amplitudes using

$$
A_{1}=\mu_{\mathrm{c}}\left(A_{2} A_{3}\right) \text {, }
$$

where $\mu_{\mathrm{c}}$ is defined as a coupling coefficient [4, 5]. The coupling coefficient $\mu_{\mathrm{c}}$ in equation 3 represents the strength of non-linearity of pulsation modes in a star and thus how much coupling exists among the members of a 
family $[4,5]$. Therefore, the testable prediction for resonant mode coupling between a child and two parent modes in a $\delta$ Sct star is amplitude modulation in three similaramplitude modes resulting in large values of $\mu_{\mathrm{c}}[4,5]$.

\section{Searching for amplitude modulation in Kepler $\delta$ Sct stars}

An ensemble of $983 \delta$ Sct stars was comprised by selecting Kepler targets that met all of the following criteria:

1. $6400 \leq T_{\text {eff }} \leq 10000 \mathrm{~K}$ in the $\mathrm{KIC}$;

2. Observed continuously from Q0 (or Q1) to Q17;

3. Contain peaks between $4 \leq v \leq 24 \mathrm{~d}^{-1}$ with amplitudes greater than $0.10 \mathrm{mmag}$.

\subsection{Identifying amplitude modulation}

A tracking routine was used to determine amplitude and phase variations (at fixed frequency) for a maximum of 12 peaks in the amplitude spectrum using the 4-yr data set for each $\delta$ Sct star. The main motivation of using 12 peaks was to identify the dominant (changes in) pulsational behaviour in each star. For a star that pulsates in only a few modes, extracting 12 peaks was more than sufficient. On the other hand, for a star that pulsates in dozens of modes and has hundreds of combination frequencies in its amplitude spectrum, 12 peaks may not be enough to fully disentangle the star, but does provide vital information on the most dominant behaviour. Note that only peaks with amplitudes greater than 0.10 mmag were extracted in this analysis, so fewer than the maximum number of 12 peaks can be extracted for a star.

After extracting the frequencies by sequentially prewhitening a star's amplitude spectrum, the frequencies were optimised using a simultaneous multi-frequency nonlinear least-squares fit to the 4-yr data set, ensuring the highest possible frequency and amplitude precision were obtained. The data set of each star was divided into 30 time bins, each $100 \mathrm{~d}$ in length (except the last bin) with a 50-d overlap, and amplitude and phase were optimised at fixed frequency using linear least-squares in each bin for each peak. This approach has previously been used to study the amplitude and phase variability in the $\delta$ Sct star KIC 7106205 [2]. The amplitudes and phases of each time bin for all extracted peaks were plotted against time in what are termed tracking plots [4], with the amplitude spectrum and tracking plot for KIC 7106205 given in the top and middle panels of figure 1 , respectively, as an example.

\subsection{Defining significant amplitude modulation}

A mean amplitude was calculated from the time bins for each peak and any bins that were more than $\pm 5 \sigma$ in amplitude away from this mean were flagged. The definition of significant amplitude modulation for each peak was defined as at least 15 (i.e., half) of its amplitude bins lying
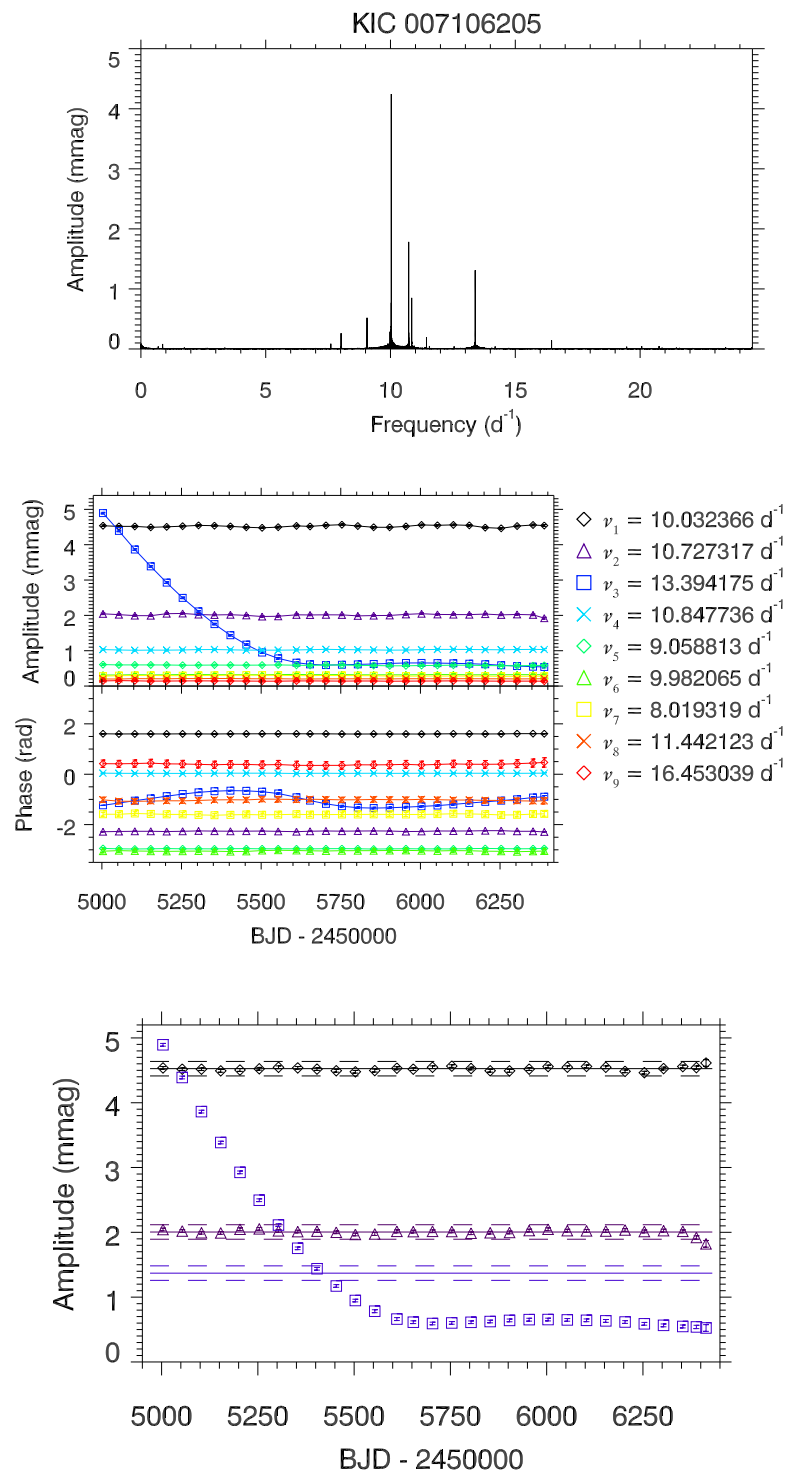

Figure 1: The top panel is the amplitude spectrum of the $\delta$ Sct star KIC 7106205, calculated using 4 yr of Kepler data. The middle panel is the amplitude and phase tracking plot for the nine significant peaks in KIC 7106205. The bottom panel is a demonstration of how a peak is defined as having significant amplitude modulation if 15 (half) of its bins lie outside $\pm 5 \sigma$ of the mean amplitude, where the solid and dashed lines represent the mean amplitude and its $\pm 5 \sigma$ significance interval, respectively, for each series of time bins.

more than $\pm 5 \sigma$ from its mean value [4]. This is demonstrated graphically for the three highest-amplitude pulsation modes in the $\delta$ Sct star KIC 7106205 in the bottom panel of figure 1. Each $\delta$ Sct star in the ensemble of 983 stars was classified as either an AMod (Amplitude Modulated) star if at least a single peak exhibited significant amplitude modulation, or a NoMod (No Modulation) star if all the extracted peaks were constant in amplitude [4]. 


\section{Results}

In this section, the results for a thorough search for amplitude modulation using $983 \delta$ Sct stars observed continuously by the Kepler Space Telescope for $4 \mathrm{yr}$ are presented. The analysis of amplitude modulation in the ensemble of $983 \delta$ Sct stars has been compiled into a single catalogue [4], which is available online as a $\mathrm{PDF}^{1}$.

\subsection{Constant pulsation mode amplitudes: NoMod stars}

An example of a $\delta$ Sct star with constant pulsation mode amplitudes (i.e., a NoMod star) is KIC 2304168, which has been previously studied using Kepler data, asteroseismically modelled, and determined to pulsate in the fundamental and first overtone radial modes [1]. The amplitude spectrum and tracking plot of KIC 2304168 are shown in the top and bottom panels of figure 2, respectively. KIC 2304168 has an effective temperature of $T_{\text {eff }}=7220 \pm 270 \mathrm{~K}$ and a surface gravity of $\log g=$ $3.67 \pm 0.19$ (cgs) [7], which are consistent with the results from stellar modelling [1]. The identification of radial pulsation modes in this star is also indicated by the period ratio of $v_{1}=8.107739 \mathrm{~d}^{-1}$ and $v_{2}=10.495495 \mathrm{~d}^{-1}$ being 0.7725 , which is predicted by stellar models [9]. Therefore, the case study of KIC 2304168 demonstrates that it is possible for $\delta$ Sct stars to be coherent pulsators with constant pulsation mode amplitudes over a time scale of 4 yr.

\subsection{Variable pulsation mode amplitudes: AMod stars}

An archetypal example of amplitude modulation in a $\delta$ Sct star is KIC 7106205 [2, 3]. This star was discovered to contain only a single pulsation mode that varied significantly in amplitude, whilst all other pulsation modes stayed constant in amplitude and phase [2]. Later, groundbased data from WASP were used to extend the study of amplitude modulation $2 \mathrm{yr}$ prior to the launch of the $K e$ pler Space Telescope [3]. The amplitude spectrum and tracking plot for KIC 7106205 are shown in the top and middle panels of figure 1, respectively, which demonstrate that the visible pulsation energy budget is not conserved in this $\delta$ Sct star.

Another example of an AMod star within the ensemble of $983 \delta$ Sct stars is KIC 4733344, with its amplitude spectrum and tracking plot shown in the top and bottom panels of figure 3, respectively. KIC 4733344 has an effective temperature of $T_{\text {eff }}=7210 \pm 260 \mathrm{~K}$ and a surface gravity of $\log g=3.50 \pm 0.23$ (cgs) [7]. KIC 4733344 pulsates with three relatively high-amplitude pulsation modes that exhibit significant amplitude modulation, which are labelled as $v_{1}, v_{2}$ and $v_{3}$ in order of decreasing amplitude in figure 3. Frequency analysis of KIC 4733344 revealed that its pulsation mode frequencies $v_{2}=7.226764 \mathrm{~d}^{-1}$ and $v_{3}=9.412445 \mathrm{~d}^{-1}$ have a period ratio of 0.7678 , which

\footnotetext{
${ }^{1}$ http://uclandata.uclan.ac.uk/42/
}
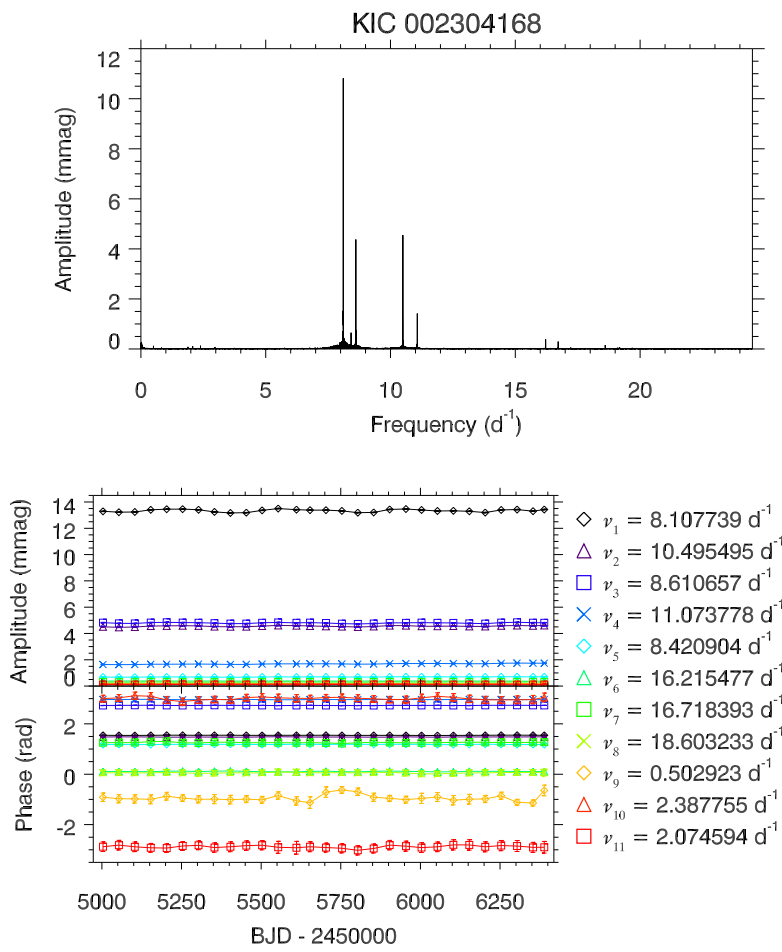

Figure 2: The amplitude spectrum and the tracking plot for the NoMod $\delta$ Sct star KIC 2304168 are shown in the top and bottom panels, respectively. There are eleven significant peaks in the amplitude spectrum of KIC 2304168, which all show constant amplitudes over the 4-yr Kepler data set using the $\pm 5 \sigma$ definition of significant amplitude modulation.

is typically associated with the fundamental and first overtone radial modes [9].

In this section, two $\delta$ Sct stars with similar $T_{\text {eff }}$ and $\log g$ values have been presented, specifically the NoMod $\delta$ Sct star KIC 2304168 and the AMod $\delta$ Sct star KIC 4733344. However, it is not immediately obvious why these two stars can be similar in their stellar parameters, and yet be so different in their observed pulsational behaviour. Further work is needed to understand the diverse range of observed pulsations in $\delta$ Sct stars, and especially the mechanism that causes amplitude modulation.

\subsection{Amplitude modulation across the classical instability strip}

Using the described methodology, amplitude modulation was discovered to be common among $\delta$ Sct stars, with 603 stars (61.3 per cent of the $983 \delta$ Sct stars in the ensemble) being classified as AMod [4]. However, amplitude modulation is not ubiquitous in $\delta$ Sct stars and further work is needed to address why this is the case. The distribution of AMod and NoMod stars on a $T_{\text {eff }}-\log g$ diagram is shown in figure 4, which shows that amplitude modulation is not restricted to a specific region within the classical instability strip in the HR diagram, and is unlikely to be related to these stellar parameters. 

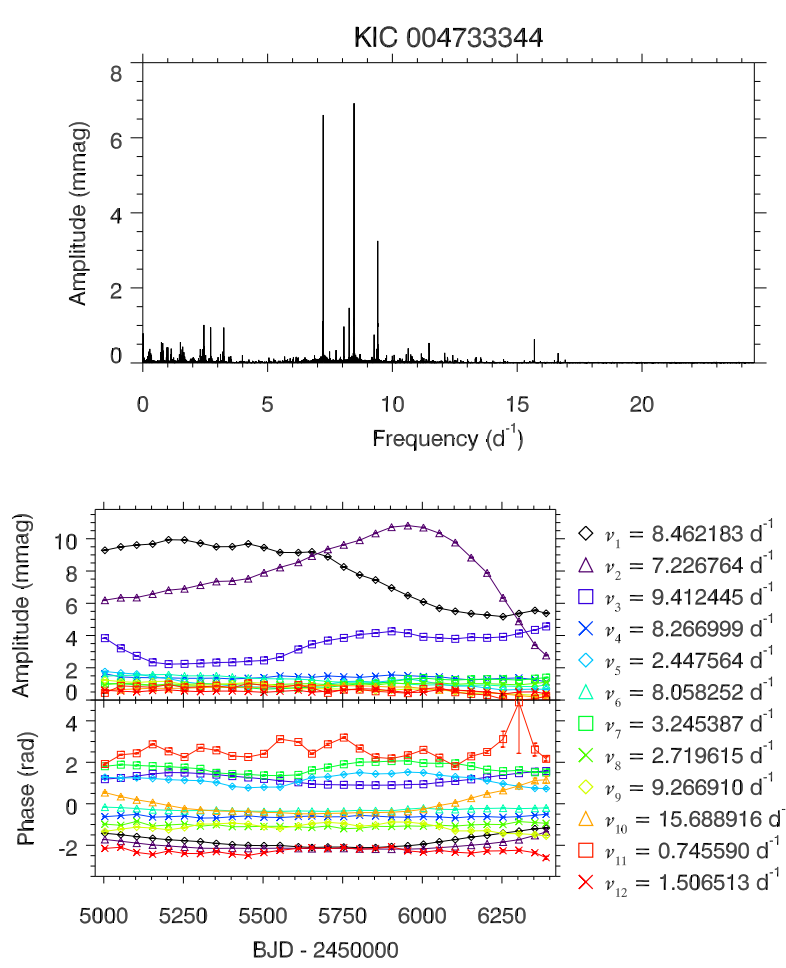

Figure 3: The amplitude spectrum and tracking plot for the AMod $\delta$ Sct star KIC 4733344 are shown in the top and bottom panels, respectively. There are many peaks in the amplitude spectrum of KIC 4733344, with only the twelve highest-amplitude peaks shown in the tracking plot.

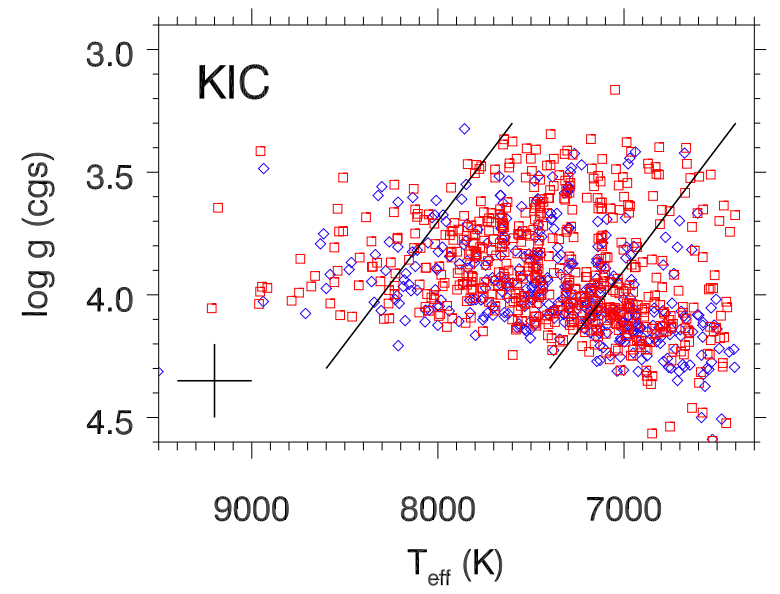

Figure 4: The ensemble of $983 \delta$ Sct stars in a $T_{\text {eff }}-\log g$ diagram using KIC parameters, with each star's location given as a red square or a blue diamond for if it is AMod or NoMod, respectively. The cross in the bottom-left corner of the figure represents the typical uncertainty for each point, and the solid lines are the observed blue and red edges of the classical instability strip [8].

\section{Conclusions}

The Kepler data set provided extremely high frequency and amplitude precision, which was used to track amplitude and phase at fixed frequency in 100-d bins with a 50-d overlap, for a maximum number of 12 peaks with amplitudes greater that $0.10 \mathrm{mmag}$ in $983 \delta \mathrm{Sct}$ stars [4]. These results were collated into an amplitude modulation catalogue and a selection of case study stars have been used to demonstrate the diversity in observed pulsational behaviour. A total of $603 \delta$ Sct stars (61.3 per cent) exhibit at least one pulsation mode that varies significantly in amplitude over $4 \mathrm{yr}$, and so amplitude modulation is common, but not ubiquitous, in $\delta$ Sct stars [4].

For many $\delta$ Sct stars, the visible pulsation mode energy is not conserved in $4 \mathrm{yr}$ of Kepler observations [4]. For example, the $\delta$ Sct star KIC 7106205 has only a single variable pulsation mode, which is shown in figure 1, and the $\delta$ Sct star KIC 4733344 contains many variable pulsation modes, which is shown in figure 3 . Amplitude modulation in $\delta$ Sct stars may be caused by resonant mode coupling of parent modes to a high-degree child mode or internal g modes [6], but this is difficult to test using Kepler data [4]. This work has demonstrated that non-linearity is important when studying $\delta$ Sct stars on time scales as short as $4 \mathrm{yr}$ [4].

The catalogue of $983 \delta$ Sct stars using $4 \mathrm{yr}$ of Kepler data demonstrates that observations spanning years (and longer) are often needed to study and resolve pulsational behaviour in these stars [4], which will be useful for comparison purposes when studying observations of $\delta$ Sct stars from K2, TESS and PLATO. Eventually, these missions will observe a large area of the sky but only for a short length of time. Therefore, Kepler may represent the best data set for studying $\delta$ Sct stars as 4 yr of continuous observations may not be surpassed for some time.

\section{References}

[1] Balona L. A., Dziembowski W. A., MNRAS 417, 591 (2011)

[2] Bowman D. M., Kurtz D. W., MNRAS 444, 1909 (2014)

[3] Bowman D. M., Holdsworth D. L., Kurtz D. W., MNRAS 449, 1004 (2015)

[4] Bowman D. M., Kurtz D. W., Breger M., Murphy S. J., Holdsworth D. L., MNRAS 460, 1970 (2016)

[5] Breger M., Montgomery M. H., ApJ 783, 89 (2014)

[6] Dziembowski W., Acta Astronomica 32, 147 (1982)

[7] Huber D., et al., ApJS 211, 2 (2014)

[8] Rodríguez E., Breger M., A\&A 366, 178 (2001)

[9] Stellingwerf R. F., ApJ 227, 935 (1979)

[10] Uytterhoeven K. et al., A\&A 534, A125 (2011)

[11] Wu Y., MNRAS 323, 248 (2001) 